\section{SAT0133 PREDICTORS OF PAIN AND ITS CHANGE OVER TIME IN FIBROMYALGIA}

${ }^{1} \mathrm{PH}$ Dessein, 'AE Stanwix, ${ }^{2} \mathrm{Z}$ Moomal. 'Rheumatology, Witwatersand University/ Johannesburg, Johannesburg; '2Statistics, National Research Foundation, Pretoria, South Africa

\subsection{6/annrheumdis-2001.592}

Background Widespread pain is the central feature of fibromyalgia (FM). The role of physical as opposed to psychological mechanisms in FM pain remains a matter of controversy.

Objectives In the present study, we evaluated the dependency of FM pain on other FM clinical features as assessed by the Fibromyalgia Impact Questionnaire (FIQ).

Methods Forty two consecutive patients, who had been followed up for at least 2 months, were enrolled. Their mean (SD, range) age, disease duration and follow up duration were 42 (12, 1578), $8(11,0.3-45)$ and $2.9(2.2,0.28-8.6)$ years, respectively. They completed FIQ scores as applied to their health status at enrolment in the clinic and at the time of the study. Statistical analyses were done by simple linear regression analyses and oneway analysis of variance. Results were expressed as mean (SD) and a $\mathrm{p}<0.05$ was considered significant.

Results The pain visual analogue scale was 8.9 (1.3) at enrolment versus $2.4(1.9)$ at the time of the study $(\mathrm{p}<0.001)$. At enrolment, pain was predicted by morning tiredness $(\mathrm{p}<$ 0.001), FM symptom interference with work $(\mathrm{p}<0.001)$ and stiffness $(p=0.001)$. At the time of the study, pain was predicted by tiredness $(p<0.001)$, morning tiredness $(p<0.001)$, FM symptom interference with work $(\mathrm{p}=0.003)$ and stiffness $(p=0.03)$. Improvement in pain was predicted by pain at enrolment ( $\mathrm{p}=0.007)$ (and by no other baseline FIQ scores) and improvements in tiredness $(\mathrm{p}<0.001)$, morning tiredness $(\mathrm{p}<$ $0.001), F M$ symptom interference with work $(\mathrm{p}<0.001)$ and stiffness $(\mathrm{p}=0.002)$.

Conclusion Pain and fatigue were strongly interrelated in our FM patients. The physical symptoms fatigue, stiffness and FM symptom interference with work consistently predicted FM pain and its change over time. In contrast, the psychological variables anxiety and depression, as assessed by the FIQ, did not relate to FM pain.

\section{REFERENCES}

1 Goldenberg DL. Arch Intern Med. 1999;159:777-85

2 Dessein $\mathrm{PH}$, et al. Pain Rev. 1997:4:79-88

3 Burckhardt CS, et al. J Rheumatol. 1991;18:728-37

\section{SAT0134 PATIENT PERCEIVED BENEFITS AND SIDE EFFECTS OF THERAPEUTIC INTERVENTIONS IN FIBROMYALGIA}

${ }^{1} \mathrm{PH}$ Dessein, ${ }^{1} \mathrm{AE}$ Stanwix, ${ }^{2} \mathrm{Z}$ Moomal. 'Rheumatology, Witwatersrand University/ Johannesburg Hospital, Johannesburg; ${ }^{2}$ Statistics, National Research Foundation, Pretoria, South Africa

\subsection{6/annrheumdis-2001.593}

Background The efficacy of treatment programs in fibromyalgia (FM) as currently used in practice is unsatisfactory and more innovative and effective therapies need to be developed.

Objectives The present study comprises part of an audit of a rheumatology clinic in which central sensitisation and neuroendocrine deficiencies were considered in the evaluation and management of FM.

Methods Forty two consecutive female FM (22 primary; 20 sary) patients, who had been followed up for at least 2 months, were enrolled. Their mean (SD, range) age was 42 (12, 15-78), disease duration $8(11,0.3-45)$ and follow up duration 2.9 (2.2, 0.28-8.6) years, respectively. Patients completed Fibromyalgia Impact Questionnaires (FIQ) and reported on the benefits and side effects of each intervention. Results were expressed as mean (SD) where appropriate.

Results The total FIQ improved from 74.1 (11.9) to 21.6 (13.2) $(\mathrm{p}<0.001) . \mathrm{N}$ users and $\%$ patients of users benefiting, experiencing side effects and discontinuing treatment for the different interventions were: (1) dehydroepiandrosterone: 33, 97, 6, 6; (2) IM methylprednisolone (120 mg): 33, 91, 9, 21 (duration of benefit: 2.4 (2.6), range 0-13 months); (3) exercise: 30, 100, 3, 0 ; (4) diet: 30, 100, 0, 0; (5) NSAID: 26, 69, 31, 50; (6) hydrocortisone: $26,73,35,31$; (7) clonazepam: 19, 89, 11, 21; (8) clonidine: 18, 90, 0, 22; (9) tri/tetracyclics: 18, 89, 44, 39; (10) trazodone: $16,87,25,13$; (11) subcutaneous sterile water: 16 , $87,0, \mathrm{~N} / \mathrm{A}$ (duration of benefit: 2.5 (3.1), range 0-12 months); (12) pemoline: 14, 64, 21, 71; (13) fludrocortisone: 13, 85, 8, 38 ; (14) chloroquine (in secondary fibromyalgia only): 12, 100, 0,8 . More detailed results will be presented. Each FIQ score improved more in secondary as compared to primary FM and these differences reached significance for tiredness and morning tiredness $(p<0.04)$. After controlling for the use of chloroquine, the respective differences disappeared.

Conclusion Although none of our patients were involved in disability claims and only $4(10 \%)$ were not working because of their disease, our results suggest that an individualised and multimodal management approach is associated with a marked improvement in health status in FM. Also, the potential role of chloroquine in primary FM deserves evaluation.

\section{REFERENCES}

1 Wolfe F, et al. Arthritis Rheum. 1997;40:1571-9

2 Dessein $\mathrm{PH}$, et al. Pain 2000;86:213-15

\section{SAT0135 ASSOCIATION BETWEEN HEPATITIS C VIRUS ANTIBODY, HEPATITIS B VIRUS ANTIGEN AND FIBROMIYALGIA}

A Gür, M Karakoç, MF Geyik, K Nas, R Çevik, AJ Saraç, S Em, F Erdogan. Physical Therapy and Rehabilitation, Infection Disease, Dicle University, Diyarbakyr, Turkey

10.1136/annrheumdis-2001.594

Background Although Hepatitis B virus and hepatitis C virus infections have been associated with several autoantibodies, the relationship between $\mathrm{HCV}, \mathrm{HBV}$ infections and autoimmun response and autoimmune diseases remains unknown. In the literature there are suspectible results related to antibody positivity against $\mathrm{HCV}$ and antigen positivity $\mathrm{HBV}$ in cases with fibromiyalgia syndrome and rheumatoid arthritis.

Objectives The objective was to determine whether there might be association between HBs-Ag, anti-HCV and fibromyalgia.

Methods We determined the prevalence of Anti-HCV, in 51 fibromiyalgia patients, in comparison 35 rheumatoid arthritis patients from the Dicle University Hospital.

Results Among 51 patients with fibromyalgia, we detected 10 patients (19.6\%) with HBs-Ag and 1 (1.96\%) anti-HCV antibody, whereas we found no HBs-Ag and anti-HCV antibody among in all of the rheumatoid arthritis cases. We found statistically significant difference when we compared the HBs-Ag positivity established in fibromiyalgia cases with those in healthy blood donors (which is found to be $4.87 \%$ in our region) and cases with rheumatoid arthritis $(p<0.05)$. Also, there was not any difference in terms of anti-HCV antibody positivity between 